\title{
Ultrasonic evaluation of movement of the diaphragm after acute cerebral infarction
}

\author{
J G Houston, A D Morris, D G Grosset, K R Lees, N McMillan, I Bone
}

\begin{abstract}
Respiratory dysfunction is an important complication of acute stroke but its mechanisms are poorly understood. Previous indirect assessments suggest that paralysis of the diaphragm occurs contralateral to the cerebral lesion. Diaphragmatic excursion was studied with real time ultrasound during quiet and deep breathing in 50 patients within 72 hours of acute stroke and 40 controls. During quiet breathing, hemidiaphragmatic movements were not significantly different between right hemispheric stroke, left hemispheric stroke, and controls. During deep inspiration, there was a significant bilateral reduction in hemidiaphragmatic excursion in patients with stroke, for both right hemispheric stroke and left hemispheric stroke when compared with controls (both $P<0.001$ ). Thus isolated hemidiaphragmatic paresis does not occur but maximal excursion of the diaphragm is reduced bilaterally in patients with acute stroke. This is a likely contributor to the respiratory dysfunction after acute stroke.
\end{abstract}

(F Neurol Neurosurg Psychiatry 1995;58:738-741)

Keywords: ultrasound; cerebral infarction; diaphragmatic movement

Department of Diagnostic Radiology

$\mathrm{J}$ G Houston

N McMillan

University

Department of

Medicine and

Therapeutics,

Western Infirmary,

Glasgow G11 6NT,

Scotland

A D Morris

D G Grosset

$\mathrm{K} R$ Lees

I Bone

Correspondence to:

Dr A D Morris, Department

of Pharmacology and

Clinical Pharmacology,

Ninewells Hospital and

DD1 9SY, Scotland.

DD1 9SY, Scotland.

1994 and in revised form

17 January 1995.

Accepted 10 February 1995
Traditionally, movement of the diaphragm is assessed by fluoroscopy. We have recently validated real time ultrasound as a reproducible, non-invasive alternative. ${ }^{4}$ We undertook the current study to determine whether hemidiaphragmatic movement is impaired in acute cerebral infarction.

\section{Methods}

Patients with acute ischaemic stroke admitted during a six month period to our acute stroke unit were studied. Prior informed consent was obtained from patient or family and the protocol received ethical approval. The inclusion criteria were (a) symptoms of anterior cerebral circulatory ischaemic stroke, (b) lifetime first ever stroke, $(c)$ no history of significant cardiorespiratory disease, (d) normal chest radiograph, and (e) ultrasound studies completed within 72 hours of stroke onset. Comatose patients and those unable to take a voluntary deep breath were excluded. Control patients recruited from a general medical ward had no respiratory or neuromuscular disease, a normal chest radiograph, and were examined before discharge after resolution of the presenting complaint.

All patients with stroke underwent nonenhanced cerebral CT (Tomoscan, 310 Phillips). Presentation was defined clinically ${ }^{5}$ and graded by the Canadian Neurological Score (CNS). ${ }^{6}$ The method of assessing diaphragmatic movement has been described in detail elsewhere. ${ }^{4}$ Briefly, the patient was examined supine after a period of 15 minutes rest with an Acuson 128 computed sonography system (Acuson, California) with a $3 \mathrm{MHz}$ sector transducer. Symmetric intercostal probe positions were chosen between the midaxillary and midclavicular lines. Both hemidiaphragms were examined in a longitudinal plane that included the maximal renal bipolar length, and craniocaudal movement of the posterior muscular crus of each hemidiaphragm was measured in five consecutive respiratory cycles of both quiet and deep respiration (fig 1). The mean between and within observer coefficients of variation for this technique are $8 \%$ and $9 \%$ respectively. All scans were performed by one investigator (JGH) and recorded on videotape; individual hemidiaphragmatic excursions were measured by a second investigator blinded to patient details (ADM). 

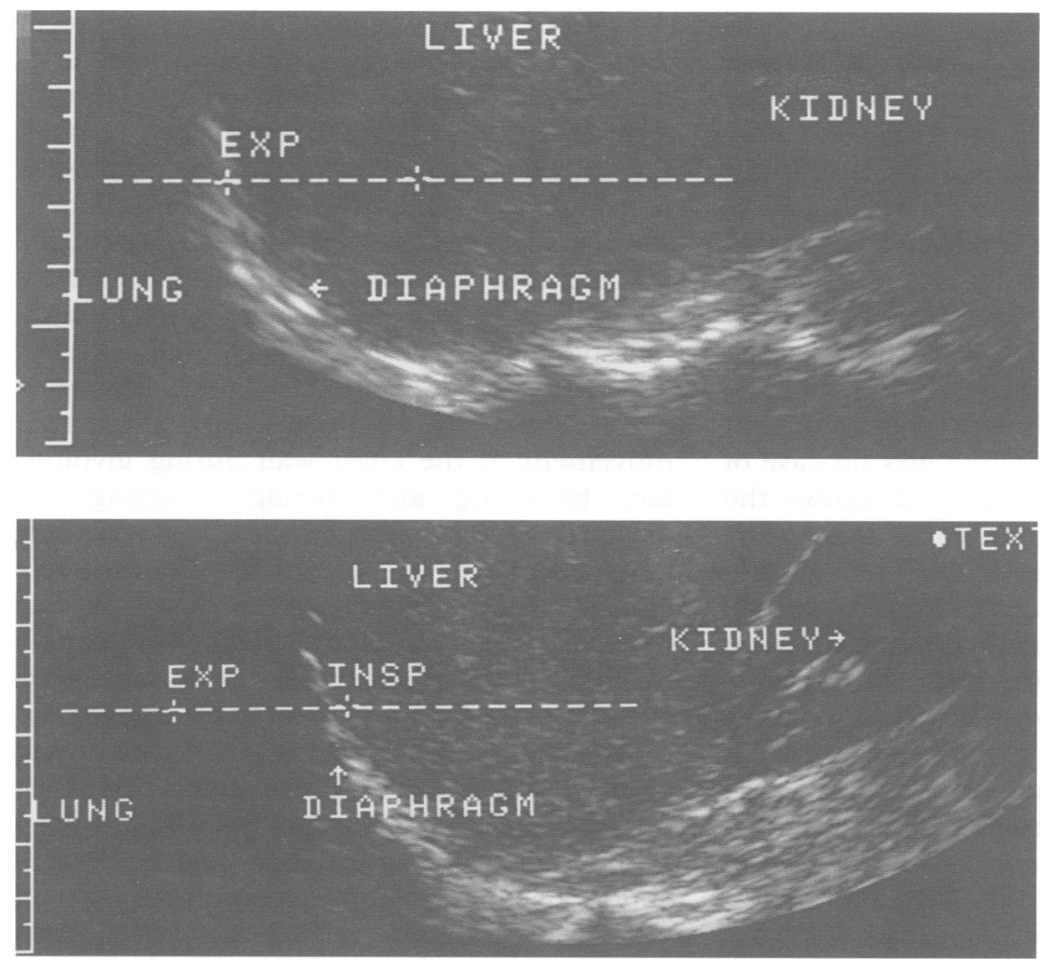

Figure 1 Craniocaudal ultrasound image of the right hemidiaphragm during expiration (top) and inspiration (bottom). Hemidiaphragmatic excursion was measured as shown $(-+----+-)$.
To assess dominance of the hemidiaphragm, the right to left ratios of diaphragmatic excursion were calculated for each subject. In a normal population, this ratio is $1 \cdot 1$ (SD $0 \cdot 2)$, consistent with the overall right hemidiaphragm dominance. ${ }^{4}$ The within group side to side differences and the between group variability of movements of the hemidiaphragm were analysed by repeated measures analysis of variance (ANOVA) with the statistical package Rummage on an ICL8988 computer. All results are expressed as means (SD).

\section{Results}

Twenty five patients with right hemispheric stroke (median age 73 (range 45-87) years), 25 patients with left hemispheric stroke (median 72 (range 43-89) years), and 40 controls (median 68 (range 43-95) years) were studied. Clinical presentation was similar in both stroke groups; in total $58 \%$ presented with a partial anterior circulation syndrome, $28 \%$ with a total anterior circulation syndrome, and $14 \%$ with a lacunar syndrome. At the time of study, patients with stroke had a neurological deficit ranging from mild weakness to total flaccid paresis; median CNS scores in the right and left hemispheric

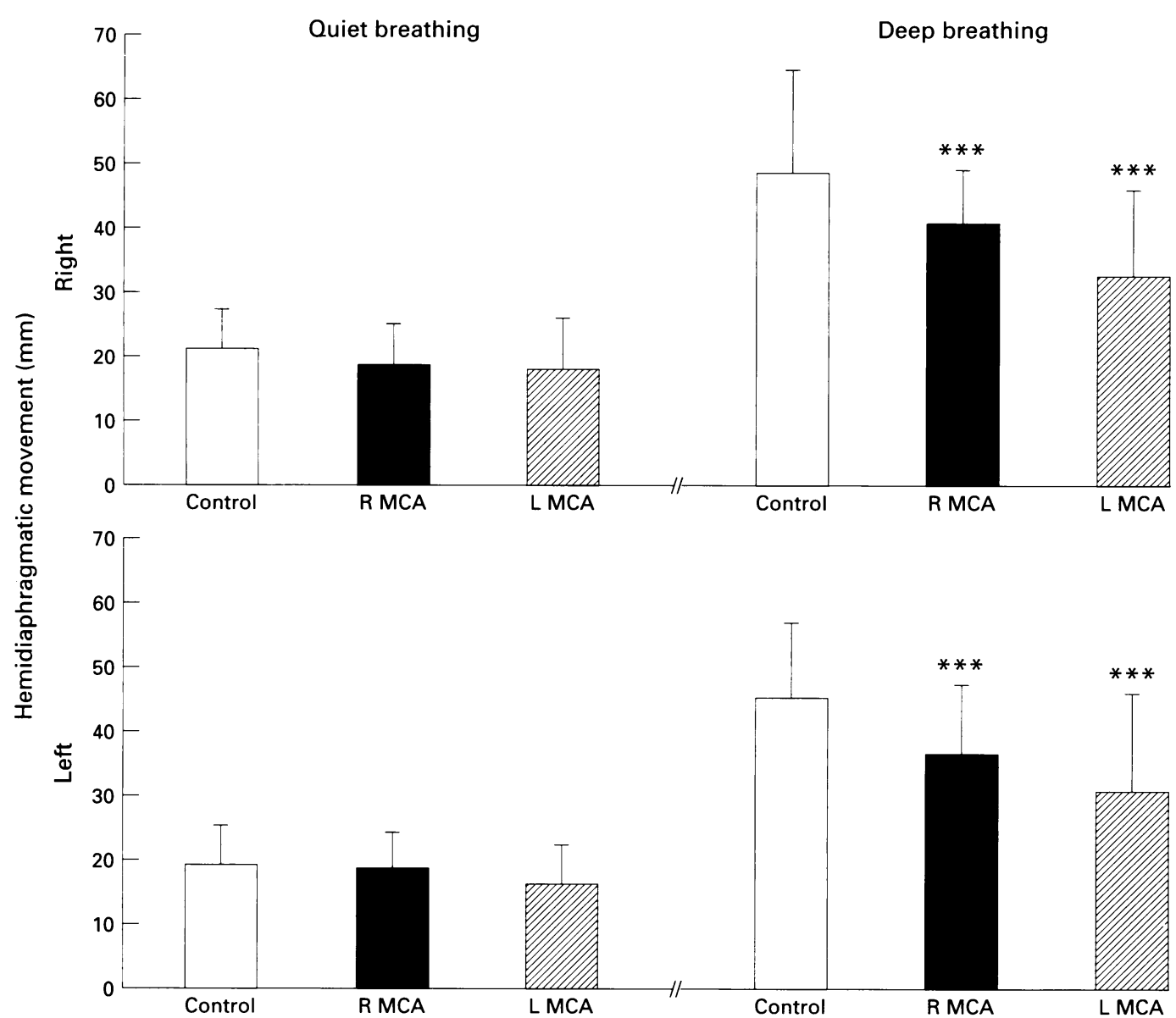

Figure 2 Hemidiaphragmatic movement during quiet and deep breathing in 50 cases of ischaemic stroke and 40 controls. There was no difference in excursion of the diaphragm during quiet breathing between the groups; during deep breathing both stroke groups had significantly reduced excursion compared with controls; $R=$ right; $L=$ left; $M C A=$ middle cerebral artery stroke. Data are means (SD); $\star \star \star P<0.001$. 
stroke groups were 40 (range 5-90) and 45 (range 10-90) respectively. There was no significant difference in the CT findings in the two groups; in total $22 \%$ of scans were normal, 30\% showed stem middle cerebral artery (MCA) infarction, 32\% branch MCA infarction and $24 \%$ lacunar infarction. Isolated hemidiaphragmatic elevation was not seen on the admission chest radiograph in any of the patients.

Adequate views of both hemidiaphragms and bilateral hemidiaphragm excursion was detected in all subjects. There was no case of paradoxical movement. Figure 2 shows the diaphragmatic excursion in each group during quiet and deep breathing. Thus during quiet breathing, hemidiaphragmatic movements were not significantly different between right hemispheric stroke, left hemispheric stroke, and controls. For example, in patients with right hemispheric stroke, right and left hemidiaphragmatic excursions were 18.7 (7) $\mathrm{mm}$ and $18(6 \cdot 2) \mathrm{mm}$ respectively compared with $21.2(6.2) \mathrm{mm}$ and $19.3(5.9) \mathrm{mm}$ respectively for controls. The $95 \%$ confidence intervals (95\% CIs) for right hemispheric stroke group $v$ controls were -1.7 to $6.8 \mathrm{~mm}$ and -1.0 to $7.3 \mathrm{~mm}$ for right and left hemidiaphragmatic excursion respectively. Similar results were obtained comparing controls with left hemispheric strokes during quiet breathing.

By contrast, during deep inspiration, there was a significant bilateral reduction in hemidiaphragmatic excursion in stroke patients, for both right hemispheric stroke (right hemidiaphragmatic movement 41.0 (9.1) $\mathrm{mm}$, left hemidiaphragmatic movement 35.8 $(11.4) \mathrm{mm}$, both $\mathrm{P}<0.001$, ANOVA), and left hemispheric stroke (right hemidiaphragmatic movement $32.7(13.8) \mathrm{mm}$, left hemidiaphragmatic movement $30.0(15.9) \mathrm{mm}$, both $P<0.001$ ), when compared with controls (right hemidiaphragmatic movement $49.0(6.3) \mathrm{mm}$, left hemidiaphragmatic movement $45 \cdot 1(12 \cdot 6) \mathrm{mm})$. Both hemidiaphragms were equally affected (fig 2). For right hemidiaphragmatic movement, the $95 \%$ CIs for control and right hemispheric strokes and control and left hemispheric strokes were 7 to $25 \mathrm{~mm}$, and 3 to $17 \mathrm{~mm}$ respectively. Similar results were obtained for left hemidiaphragmatic movement.

There was no significant difference in ratios between right or left hemisphere groups and controls. For example, in the right hemispheric stroke group the ratios were $1.0(0.5)$ and $1.2(0.3)$ during quiet and deep breathing compared with $1.2(0.4)$ and $1 \cdot 1(0 \cdot 2)$ respectively for controls. The $95 \% \mathrm{CI}$ for this finding was -0.4 to 0.1 for both groups $v$ control.

\section{Discussion}

The secondary complications of stroke will remain important even if current treatment trials identify an effective neuroprotective agent. Aetiological factors in the development of hypostatic pneumonia deserve study with the aim of improving physiotherapy and techniques of nursing care. The incidence of pneumonia may be affected in the longer term by poor cough and aspiration, because the cough mechanism is dependent on inspiratory muscle strength. ${ }^{7}$ Also, limitation of respiratory function may hinder physiotherapy and speech therapy ${ }^{89}$ impairing the extent of recovery as well as rate of progress in rehabilitation. ${ }^{10}$

Carbon dioxide rebreathing techniques in hemiparetic patients have shown symmetric movement of the chest wall during involuntary breathing and during coughing but diminished movement of the chest wall during voluntary respiration. ${ }^{11}$ Fluoroscopic studies report diminished excursion of the hemidiaphragm ipsilateral to the hemiparesis during voluntary inspiration in patients with stroke. ${ }^{12}$ Symmetric chest wall movement during involuntary breathing and diminished chest wall movement during voluntary respiration ipsilateral to the hemiparesis has been found. ${ }^{11}$ In addition, the simple finding of elevation of the ipsilateral hemidiaphragm on chest radiography is appealing. ${ }^{1}$ The normal wide variability between hemidiaphragmatic movements on fluoroscopic ${ }^{13}$ and ultrasound ${ }^{4}$ studies, however, impairs definitive conclusions in many of the prior studies. We therefore emphasise that on fluoroscopy the excursion of one hemidiaphragm must be less than half that of the other ${ }^{13}$ and on ultrasound the right to left ratio of excursion of the hemidiaphragm must lie outside the normal range $0 \cdot 5-1 \cdot 6{ }^{4}$

With these stringent criteria, we found no reduction in diaphragmatic movement during quiet respiration, but a significant bilateral reduction during deep respiration. Taken with previous valid reports, we believe that on the hemiparetic side there is impairment not only of chest wall movement but also of diaphragmatic movement, which would be expected to jointly contribute to hypostatic pneumonia and a poor cough mechanism. Partial hemidiaphragmatic dysfunction, with passive stretching of one hemidiaphragm in response to movement of the unaffected hemidiaphragm, ${ }^{7}$ is an unlikely explanation of our findings as the passive stretch should have been greater for deep breathing. Measurement of diaphragmatic length and geometry with a respitrace or magnetometer would be required to explore this further as the craniocaudal movements of one area of the diaphragm alone gives no insight into the tension developed by the diaphragm or its interaction with the rib cage or abdominal wall muscle tone which may alter lung volume.

The neurological control of respiration is complex, consisting of a central involuntary brainstem control with higher voluntary cortical inputs. We therefore limited our study to patients with first ever anterior circulation stroke, who should have intact automatic pontomedullary mechanisms. In response to the request to "breathe deeply" resultant voluntary control of respiration is activated. The 
reduction in the excursion of the hemidiaphragm was more pronounced in patients with a left hemispheric lesion, which may indicate reduced comprehension due to receptive dysphasia. A true interhemispheric difference may exist, however, as suggested by lateralisation in threshold response, with greater respiratory depression from left rather than right thalamic stimulation. ${ }^{14}$ Overall our results suggest bilateral cortical representation, with voluntary respiration mediated by fibres in the lateral pyramidal tract. ${ }^{15}$ Certainly there is evidence from other studies that some fibres are carried in a crossed spinal pathway. ${ }^{16}$ Certainly there is no evidence of ipsilateral hemidiaphragmatic paresis in patients with acute stroke in our study. Although we cannot exclude linkage below the cortical level, and a diaphragm to diaphragm reflex could explain our findings, ${ }^{7}$ under either circumstance the functional capability of diaphragmatic movement is affected as described.

In conclusion, we have excluded hemidiaphragmatic paresis contralateral to the cerebral lesion, supporting bilateral cortical representation of each hemidiaphragm. We report a bilateral reduction in voluntary hemidiaphragmatic excursion, rather than a unilateral hemidiaphragmatic paresis, that may contribute to the reduction in respiratory function in acute stroke.

The ultrasound equipment was funded in part by the Scottish Home and Health Department and the Chest Heart and Stroke Association (Scotland).
1 Santamaria J, Ruiz C. Diaphragmatic elevation in stroke. Eur Neurol 1988;28:81-3.

2 Kaldor A, Berlin I. Pneumonia, stroke and laterality (letter). Lancet 1981;i:843.

3 De Troyer A, De Beyl DZ, Thirion M. Function of the De Troyer A, De Beyl DZ, Thirion M. Function of the
respiratory muscles in acute hemiplegia. Am Rev Respir respiratory muscles in

4 Houston JG, Morris AD, Howie CA, Reid JL, McMillan N. Technical report: quantitative assessment of diaphragmatic movement - a reproducible method using ultrasound. Clin Radiol 1992;46:405-7.

5 Bamford J, Sandercock P, Dennis M, Burn J, Warlow C. Classification and natural history of clinically identifiable subtypes of cerebral infarction. Lancet 1991;337:1521-6.

6 Cote R, Hachinski VC, Shurvell BL, Norris JW, Wolfson L. The Canadian Neurological Scale: validation and reliability assessment. Neurology 1989;39:638-43.

7 Bellemare F, Bigland-Ritchie B, Woods JJ. Contractile properties of the human diaphragm in vivo. $\mathcal{f} A p p l$ properties of the human
Physiol 1986;61:1153-61.

8 Greenberg M, Edmonds J. Chronic respiratory problems in neuromyopathic disorders. Pediatr Clin North Am 1974;21:927-32.

9 Yatsu FM, De Graba TJ, Hanson J. Therapy of secondary medical complications of strokes. In: Barnett HJM, Mohr JP, Stein BM, Yatsu FM, eds. Stroke: pathophysiology diagnosis and management London: Edward Arnold. 1992:995-1004.

10 Gresham GE. Rehabilitation of the stroke survivor. In: Barnett HJM, Mohr JP, Stein BM, Yatsu FM, eds. Stroke: pathophysiology diagnosis and management. 1992:1189-201.

11 Fluck DC. Chest movements in hemiplegia. Clin Sci 1966;31:383-8.

12 Korczyn AD, Leibowitz U, Bruderman I. Involvement of the diaphragm in hemiplegia. Neurology 1969;19: 97-100.

13 Alexander C. Diaphragmatic movements and the diagnosis of diaphragmatic paralysis. Clin Radiol 1966;17: 79-83.

14 Barlow JS. Autonomic measures as electrophysiologica techniques for evaluation of the central nervous system In: Remond, ed. Handbook of electroencephalography and clinical neurophysiology. Amsterdam: Elsevier, 1973.

15 Haymaker W, Kuhlenbeck $H$. Disorders of the brainstem and its cranial nerves. In: Baker AB, Baker LH, eds. Clinical Neurology. London: Harper and Row, 1976: $1-82$.

16 Oberholzer RJ, Tofani WO. The neural control of respiration. In: Field J, Magoun HW, Hall VE, eds. Society, 1960:1111-29. 